\title{
A Retrospective Cohort Study Comparing Utilization and Costs of Biologic Therapies and JAK Inhibitor Therapy Across Four Common Inflammatory Indications in Adult US Managed Care Patients
}

Benjamin Chastek · John White - Damon Van Voorhis · Derek Tang •

Bradley S. Stolshek

Received: December 3, 2015 / Published online: March 12, 2016

(c) The Author(s) 2016. This article is published with open access at Springerlink.com

\section{ABSTRACT}

Introduction: Biologic therapies are used to treat several inflammatory diseases, including rheumatoid arthritis (RA), psoriasis (PsO), psoriatic arthritis (PsA), and ankylosing spondylitis (AS). Data from a commercial claims database were used to evaluate utilization and cost of biologic treatment for these conditions.

Methods: Data were obtained from the Optum Research Database. Patients were aged 18-63 years with diagnosis of moderate to severe RA, PsO, PsA, and/or AS and first (index) claim for biologics abatacept, adalimumab, certolizumab pegol, etanercept, golimumab, infliximab, rituximab, tocilizumab, or ustekinumab or non-biologic tofacitinib between March 1, 2011 and February 28, 2013. One-year treatment costs were based

Enhanced content To view enhanced content for this article go to www.medengine.com/Redeem/ 1A44F0606A55DF2D.

B. Chastek $(\varangle) \cdot J$. White $\cdot$ D. Van Voorhis

Optum, Eden Prairie, MN, USA

e-mail: Benjamin.Chastek@optum.com

D. Tang · B. S. Stolshek

Amgen Inc., Thousand Oaks, CA, USA on observed paid amounts and used to impute dosing. Treatment patterns (persistence, switching, discontinuing, restarting) were evaluated.

Results: Data from 20,159 patients were analyzed for index medications abatacept $(n=583), \quad$ adalimumab $\quad(n=6521)$, certolizumab pegol $(n=415)$, etanercept $(n=9116)$, golimumab $(n=231)$, infliximab $(n=1906)$, rituximab $(n=295)$, tocilizumab $(n=165), \quad$ ustekinumab $\quad(n=922), \quad$ and tofacitinib $(n=5)$. For patients with RA only, costs were lowest for tofacitinib $(\$ 18,769)$, rituximab $(\$ 19,569)$, or abatacept $(\$ 21,877)$, and ranged from $\$ 23,682$ to $\$ 30,269$ for all other medications. For patients with PsO only, costs were lowest for adalimumab $(\$ 29,186)$, etanercept $(\$ 31,212)$, and infliximab $(\$ 32,409)$ compared with ustekinumab $(\$ 53,746)$. For patients with PsA only, costs were lowest for etanercept $(\$ 26,916)$, followed by golimumab $(\$ 27,987)$ adalimumab $(\$ 28,749)$, and infliximab $(\$ 31,974)$. Costs were lowest with etanercept for RA plus PsA $(\$ 25,477)$ and for PsO plus PsA $(\$ 29,376)$, and with golimumab for AS only $(\$ 24,225)$. Across indications, annual costs were $\$ 29,521, \$ 27,488$, and 
$\$ 28,672$ for adalimumab, etanercept, and infliximab, respectively; persistence was greatest with infliximab (range 66-79\%) compared with $11-59 \%$ for all other biologics.

Conclusion: One-year treatment costs varied considerably between medications and indications. Some newly approved agents had lower costs but further research is needed to confirm these estimates as more patients are treated.

Funding: Immunex (a wholly owned subsidiary of Amgen Inc.) and Wyeth (acquired by Pfizer).

Keywords: Ankylosing spondylitis; Biologics; Costs; Inflammatory diseases; Psoriasis; Psoriatic arthritis; Rheumatoid arthritis; Utilization

\section{INTRODUCTION}

Many inflammatory conditions are severe, chronic, and disabling diseases that can manifest in joints and skin. Rheumatoid arthritis (RA), which affects approximately 1.5 million adults in the US and an estimated $0.5-1 \%$ of people in developed nations, primarily affects the synovial membrane in the joints [1]. Psoriasis (PsO) is a skin disease occurring in approximately 4.5 million adults in the US, with an estimated prevalence of $1.4-2.5 \%$ in children, adolescents, and adults in the US [2]. PsO is frequently accompanied by comorbid psoriatic arthritis (PsA), a disfiguring form of arthritis with an estimated prevalence of $11 \%$ in patients with $\mathrm{PsO}$ in the US [3]. Ankylosing spondylitis (AS), with prevalence estimated to be $30-900$ per 100,000 people in the US, is a type of arthritis that affects the spine [1]. In addition to symptoms associated with these diseases (pain and swelling of joints and plaque formation on skin), patients suffer impaired health-related quality of life $[4,5]$.

Biologic therapies that target specific components of the immune system are important for treatment of autoimmune diseases. These include agents that target tumor necrosis factor (adalimumab, certolizumab pegol, etanercept, golimumab, and infliximab), interleukin (IL)-6 receptor (tocilizumab), CD20 (rituximab), IL-12 and IL-23 (ustekinumab), and $\mathrm{T}$ cell stimulators (abatacept). A recently approved non-biologic therapy that targets JAK (tofacitinib) is also used to treat RA; this medication has a similar chemical profile, price, and target population in the product label relative to the biologics, and is therefore included in this analysis. These drugs differ in mode of administration, dosing level, dosing schedule, and whether they are approved for first-line use after non-biologic disease-modifying antirheumatic drug (DMARD) failure. Adalimumab, certolizumab pegol, etanercept, golimumab, and ustekinumab are administered as subcutaneous (SC) injections; infliximab and rituximab are administered as intravenous (IV) infusions; and tofacitinib is administered orally (PO). Abatacept, tocilizumab, and golimumab are available for both SC and IV administration.

The cost of biologic therapies is high compared to non-biologic DMARDs; evidence-based assessment of comparative costs between biologics is critical to ensure rational healthcare resource allocation. Data comparing costs across indications from the payer's perspective and an understanding of biologic dosing patterns across different populations and payers are critical for formulary decision-makers to develop evidence-based formularies. Although prior studies evaluated the comparative costs of biologics across multi-payer claims databases, 
the data used in those studies are now relatively outdated and such analyses of multi-payer data may be less applicable to specific payers. We now report the results from a biologic utilization model using more recent utilization data from claims for beneficiaries on a biologic enrolled in a health plan of a large national commercial health insurer.

\section{METHODS}

\section{Data Source}

Data for this study were obtained from the Optum Research Database, which contains medical and pharmacy claims data with linked enrollment information. For 2011, data for approximately 12.8 million individuals are available. The enrollees in the database are widely distributed across the US. This article does not contain any new studies with human or animal subjects performed by any of the authors.

\section{Study Design}

The identification period for eligible patients was from March 1, 2011 through February 28, 2013. The index date for each patient was the date of the first claim for the index biologic. Index medications included the biologics abatacept (administered IV), adalimumab (SC), certolizumab pegol (SC), etanercept (SC), golimumab (SC), infliximab (IV), rituximab (IV), tocilizumab (IV), and ustekinumab (SC), and the non-biologic tofacitinib (PO). The baseline period was the 180 days before the index date and the follow-up period was the 360 days starting on the index date. Patients were considered persistent if they did not switch from their index biologic or have a gap in therapy of at least 45 days at any time during the 1-year follow-up. Patients who were not persistent were further classified as switching, restarting, or discontinuing without switching or restarting.

\section{Patients}

To be eligible for inclusion in the study, patients had to: have a diagnosis of RA (International Classification of Diseases, Ninth Revision, Clinical Modification [ICD-9-CM] code 714.0x), PsO (ICD-9-CM code 696.1), PsA (ICD-9-CM code 696.0), and/or AS (ICD-9-CM code 720.0); have a fill or infusion for an index biologic during the identification period that was US Food and Drug Administration-approved for the diagnosed condition; have continuous enrollment during the baseline period through the follow-up period; have valid demographic information; be 18-63 years of age on index date; and have valid cost values (paid amount on claim $>\$ 0$ ) during the baseline and follow-up periods. Exclusion criteria included: a claim for $>1$ biologic of interest on the index date; diagnosis of juvenile idiopathic arthritis, Crohn's disease, ulcerative colitis, non-Hodgkin's lymphoma, or chronic lymphocytic leukemia during the 180 days prior to or on index date (other indications for these biologics); have a medical claim for SC-administered biologic or have a pharmacy claim for IV-administered biologic; receipt of a dose of a biologic that exceeded twice the approved maximum weekly dose; or receipt of SC abatacept or a medical claim for ustekinumab. Patients were assigned to study cohorts based on index medication and treatment indication. Patients were categorized as new to biologic therapy (no claim for biologic during the baseline period) or continuing on index biologic therapy ( $\geq 1$ claim during the baseline period). 


\section{Study Measures}

Demographic characteristics, biologic dose, and treatment patterns were collected. The total costs of each biologic during the 1-year follow-up period were calculated by considering the total dose of the index biologic while the patient was persistent, the total dose of all biologics after the patients switched/restarted biologic therapy (for patients who were not persistent) and the total number of administrations. The total dose of each biologic was examined after persistence through the remainder of the follow-up period, including the dose of index biologic for patients who restarted therapy. For each claim, the dose (mg) was calculated based on the observed paid amount using the following formula: claim dose = (paid amount on claim)/ (cost per unit) $\times$ (strength associated with unit price). Wholesale acquisition costs (WAC) as of January 1, 2015 were imputed to the total index and non-index biologic doses, while 2015 Medicare schedule fees were assigned to biologic administration units to generate biologic and related administration costs, respectively. Injection/infusion administration fees were based on the 2015 Medicare Physician Fee Schedule.

\section{Statistical Considerations}

Frequency and percentage were calculated for categorical patient characteristics and study outcomes, including treatment patterns. Analyses were conducted using SAS version 9.2 (SAS Institute Inc., Cary, NC, USA). The mean annual costs per treated patient were estimated by dividing the calculated total cost incurred by all patients treated with the biologic at index within each disease subcohort. No adjusted analyses were conducted, as the findings were meant to provide population-wide estimates for eligible patients included in this database. Propensity score weighting was not implemented.

\section{RESULTS}

\section{Patients}

A total of 59,436 patients were treated with an eligible biologic during the identification period and of these, 20,159 met all of the inclusion/ exclusion criteria (Fig. 1). The most commonly used index medications were etanercept (45\%), adalimumab (32\%), and infliximab (9\%; Table 1). The most common indications were RA only (47\%), PsO only (25\%), and PsA only (9\%). The mean age of the population was 47.6 years; mean age was $<45$ years for patients treated with ustekinumab and $>50$ years for patients treated with abatacept, tocilizumab, and rituximab. The majority (55-84\%) of patients were female except for patients treated with ustekinumab (46\% female). Patients treated with abatacept, adalimumab, etanercept, golimumab, infliximab, tocilizumab, and ustekinumab were most likely to have continued therapy from baseline through follow-up, and patients treated with certolizumab or rituximab were most likely to be new to their index therapy. All patients on tofacitinib were new to their index therapy (demographics/clinical characteristics at baseline not shown because of small sample size).

\section{Cost Per Treated Patient}

When examining costs among patients with RA only, costs were lowest for those treated with tofacitinib $(\$ 18,769 ; n=5)$, rituximab $(\$ 19,569$; $n=295)$, or abatacept $(\$ 21,877 ; n=583)$, 


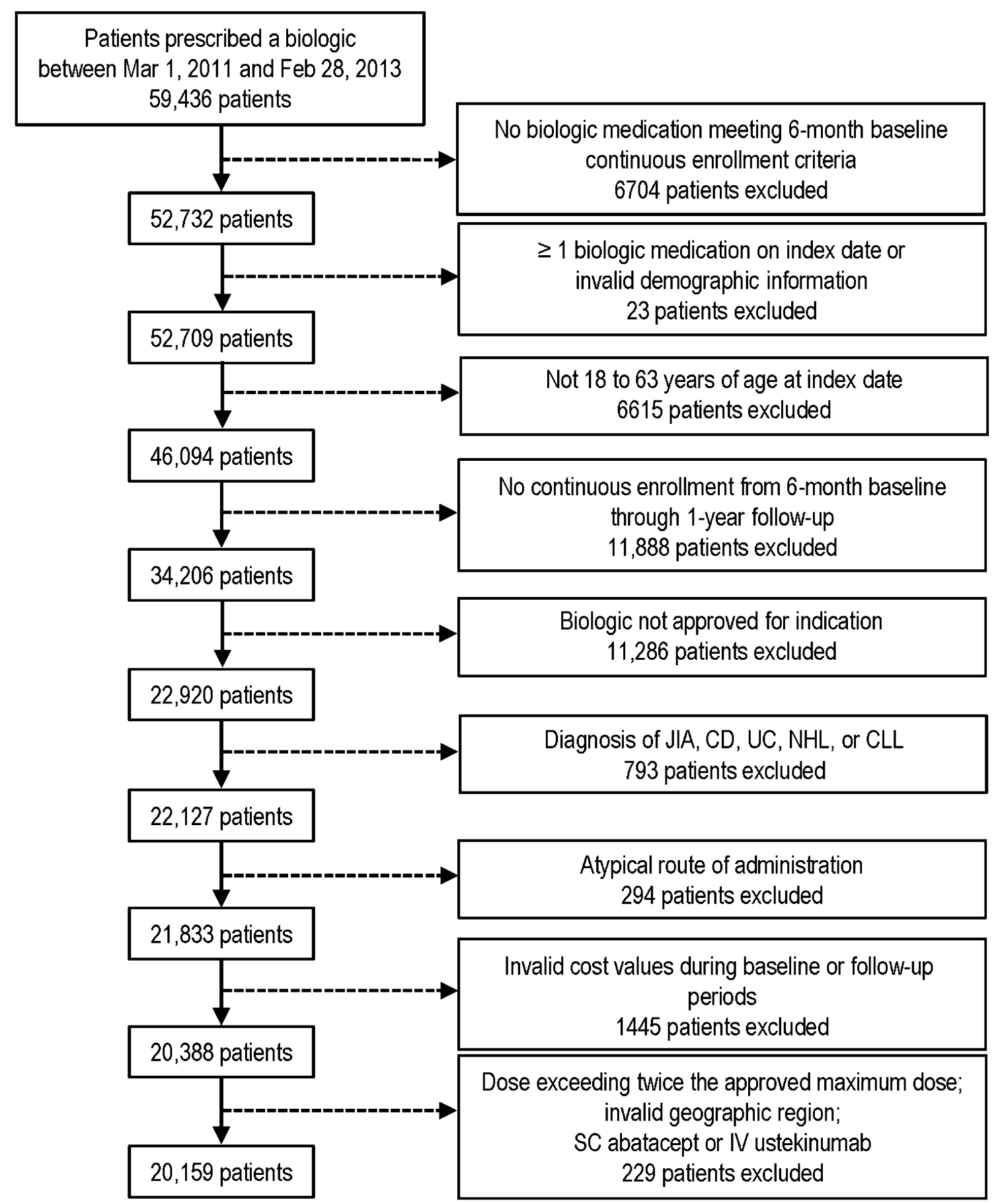

Fig. 1 Patient attrition. $C D$ Crohn's disease, $C L L$ chronic lymphocytic leukemia, $I V$ intravenous, $J I A$ juvenile idiopathic arthritis, $N H L$ non-Hodgkin's lymphoma, $S C$ subcutaneous, $U C$ ulcerative colitis

whereas patients treated with tocilizumab, etanercept, certolizumab, infliximab, golimumab, and adalimumab had average costs ranging from $\$ 23,682$ to $\$ 30,269$ $(n \quad$ range $=165-9116) \quad($ Table 2$) . \quad$ Among patients diagnosed with $\mathrm{PsO}$ only, those 


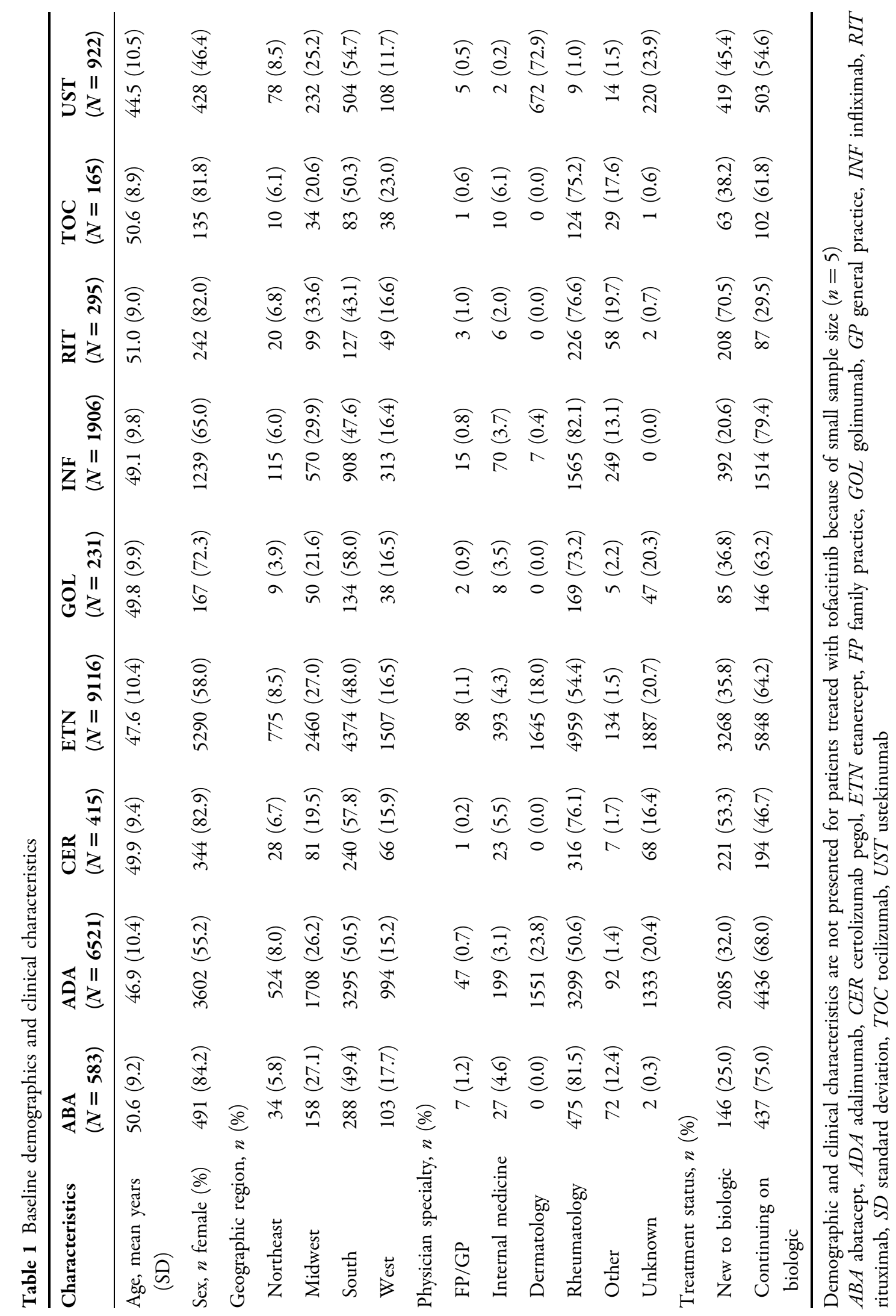




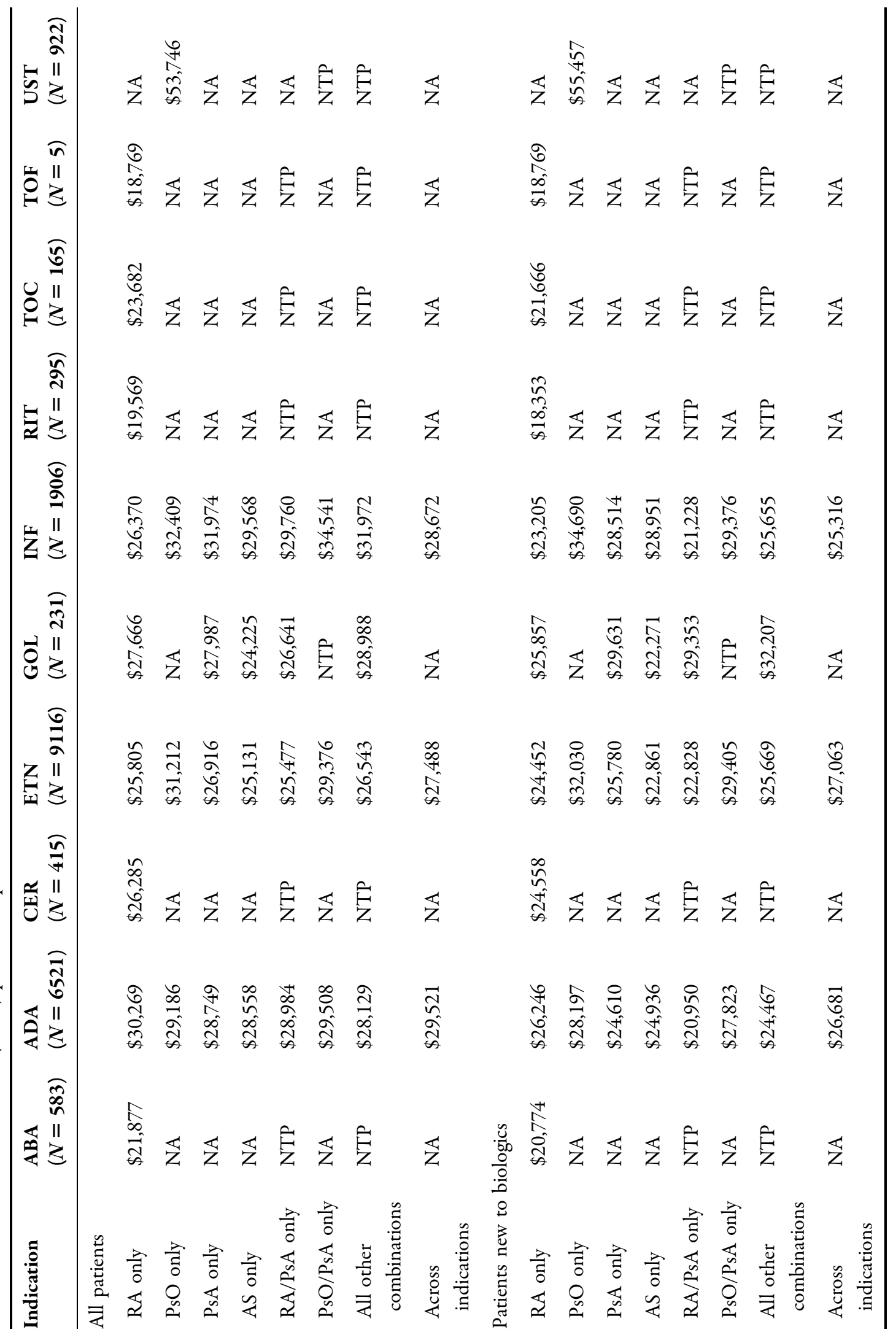




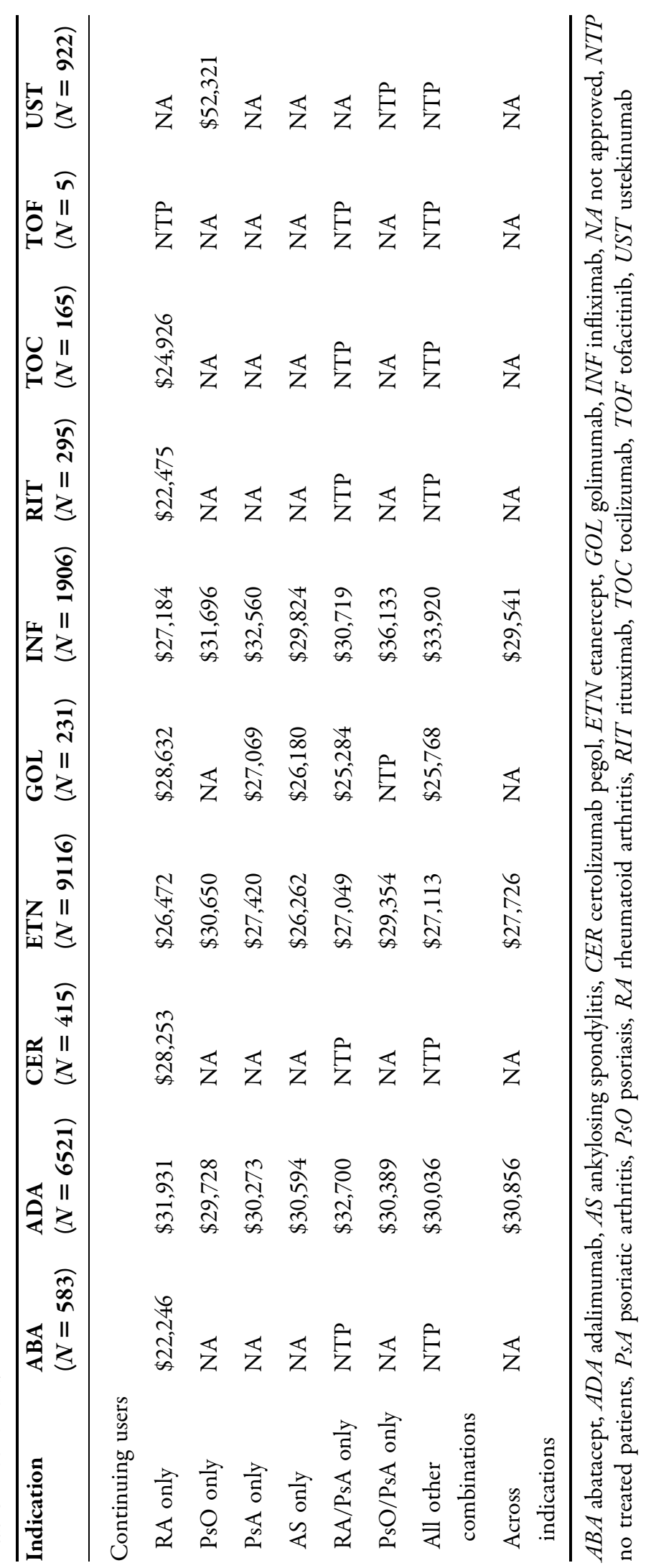


treated with ustekinumab had the greatest average annual biologic costs $(\$ 53,746$; $n=922)$ compared to adalimumab $(\$ 29,186$; $n=6521)$, etanercept $(\$ 31,212 ; n=9116)$, and infliximab $(\$ 32,409 ; n=1906)$. For patients diagnosed with PsA only, costs were lowest among those treated with etanercept $(\$ 26,916$; $n=231)$ followed by golimumab $(\$ 27,987$; $n=9116)$, adalimumab $(\$ 28,749 ; n=6521)$, and infliximab $(\$ 31,974 ; n=1906)$.

Three medications (adalimumab, etanercept, and infliximab) were approved for the treatment of all four conditions. Across indications, annual costs were $\$ 29,521$, $\$ 27,488$, and $\$ 28,672$ for adalimumab, etanercept, and infliximab, respectively. Within those 3 medication cohorts costs ranged from $\$ 25,131$ to $\$ 31,212$ for etanercept, $\$ 28,129$ to $\$ 30,269$ for adalimumab, and $\$ 26,370$ to $\$ 34,541$ for infliximab. For etanercept and infliximab, costs were greatest among patients with $\mathrm{PsO}$ only or PsO/PsA, whereas patients treated with adalimumab had the greatest costs among those diagnosed with RA only. Within each of the indications, except PsO only, the lowest cost was observed among patients treated with etanercept; for patients with $\mathrm{PsO}$ only, the lowest cost was observed among patients treated with adalimumab.

\section{Treatment Patterns}

Across all patients, persistence was greatest among patients treated with infliximab (66-79\%) compared to $41-59 \%$ for all other medications except golimumab and tofacitinib (Table 3). Patients treated with golimumab had persistence rates of $11-25 \%$ due in large part to higher rates of switching (50-76\%). Persistence rates were generally $15-25 \%$ higher among patients continuing on their index therapy compared to those who were new to therapy.

\section{DISCUSSION}

This was a retrospective analysis of total annual cost of biologic treatment and biologic utilization among patients diagnosed with at least one of four autoimmune conditions: RA, PsO, PsA, or AS. The most commonly used medications were etanercept, adalimumab, and infliximab, which is consistent with previous findings [6-8]. Notably, the most common medications had been approved longer than the other biologics included in the study. While most of the patients were considered to be continuing on existing biologic therapies, a few medication cohorts (rituximab, certolizumab, and tofacitinib) were more often new users.

The findings of this study indicate that there is a large amount of variability in biologic cost both within and between condition cohorts, which may originate from small sample sizes in some cases. For example, among patients treated for RA, costs ranged from $\$ 18,769$ for treatment with tofacitinib to $\$ 30,269$ for adalimumab. Even after excluding the very small cohort of patients treated with tofacitinib $(n=5)$ and rituximab, which has a unique dosing schedule, results still ranged from $\$ 21,877$ to $\$ 30,269$, which represents a difference of $38 \%$. The greatest variability was observed among patients treated for PsO only. Ustekinumab had the greatest cost $(\$ 53,746)$, which was $66 \%$ more than the cost of infliximab, $72 \%$ more than the cost of etanercept, and $84 \%$ more than the cost of adalimumab. Another factor that seems to be associated with increased cost is whether the patient is new to therapy at index, or continuing therapy from baseline to follow-up. 


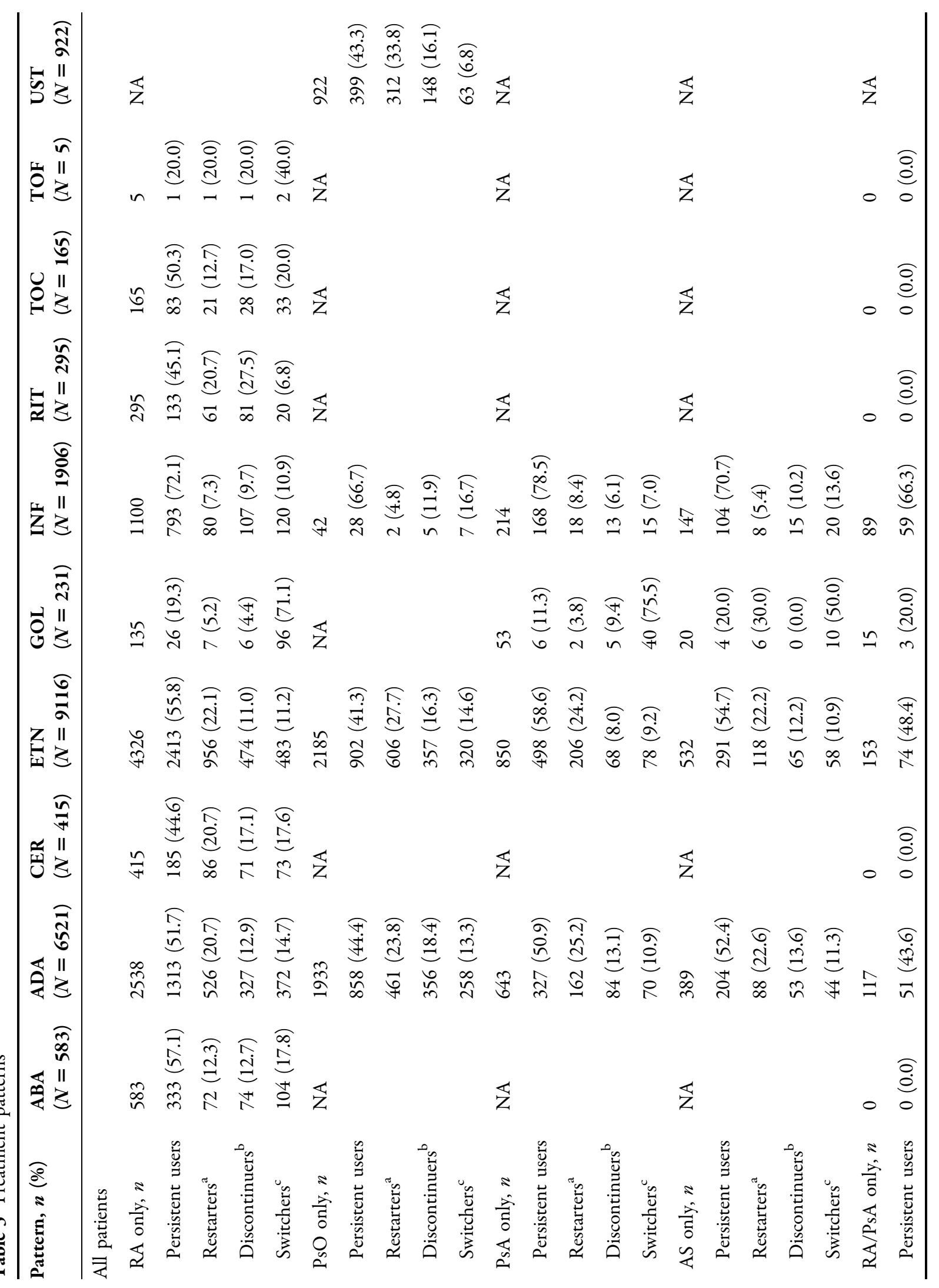




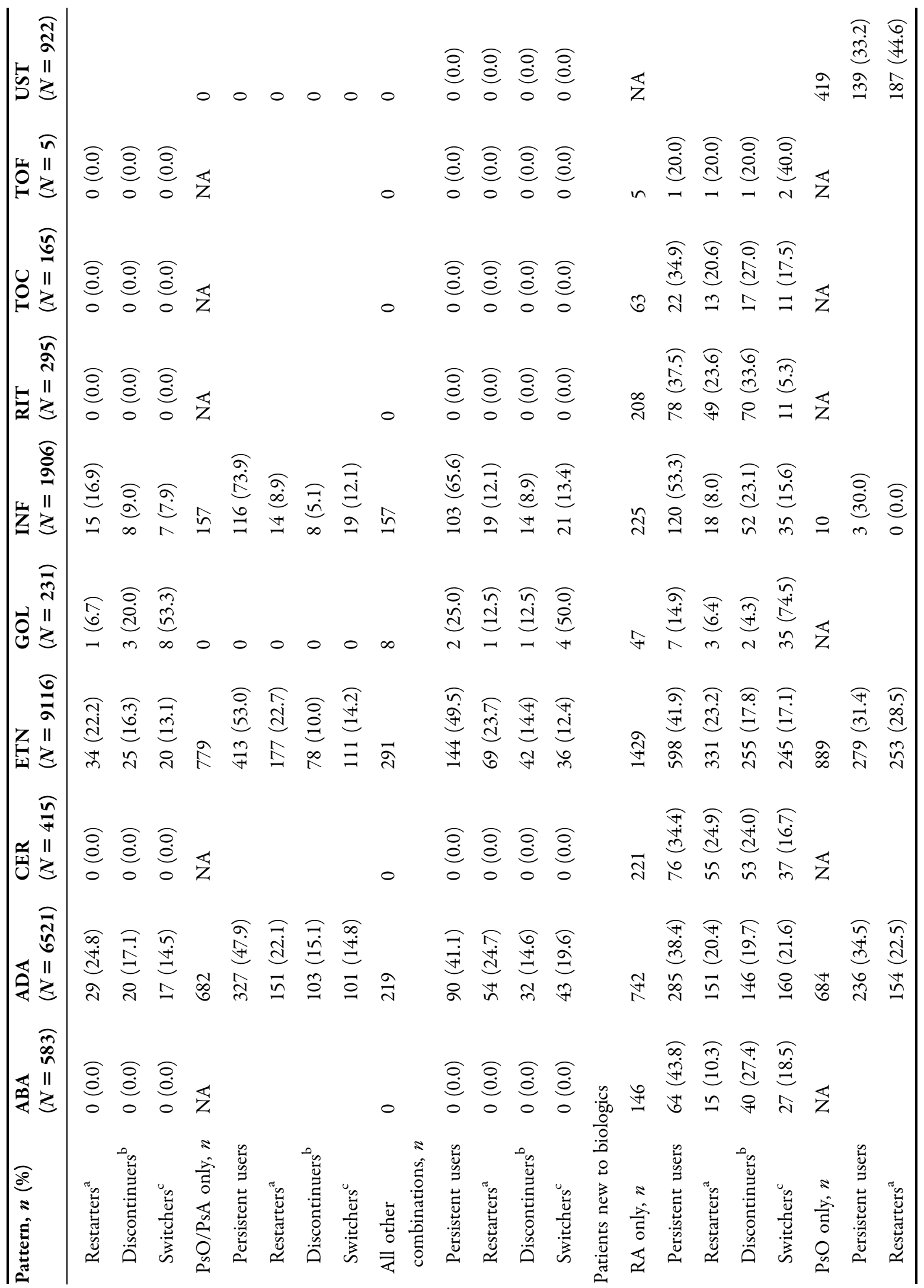




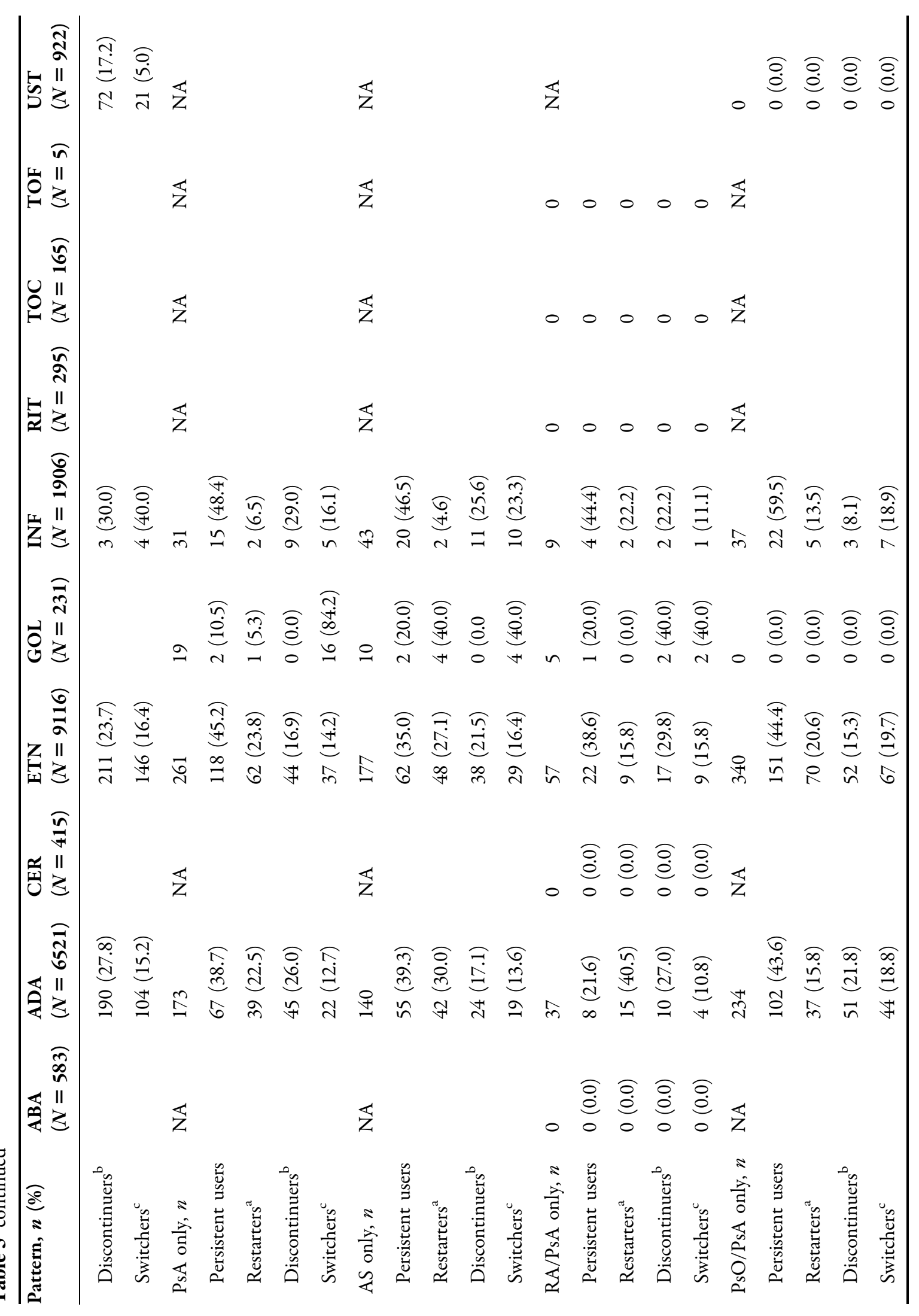




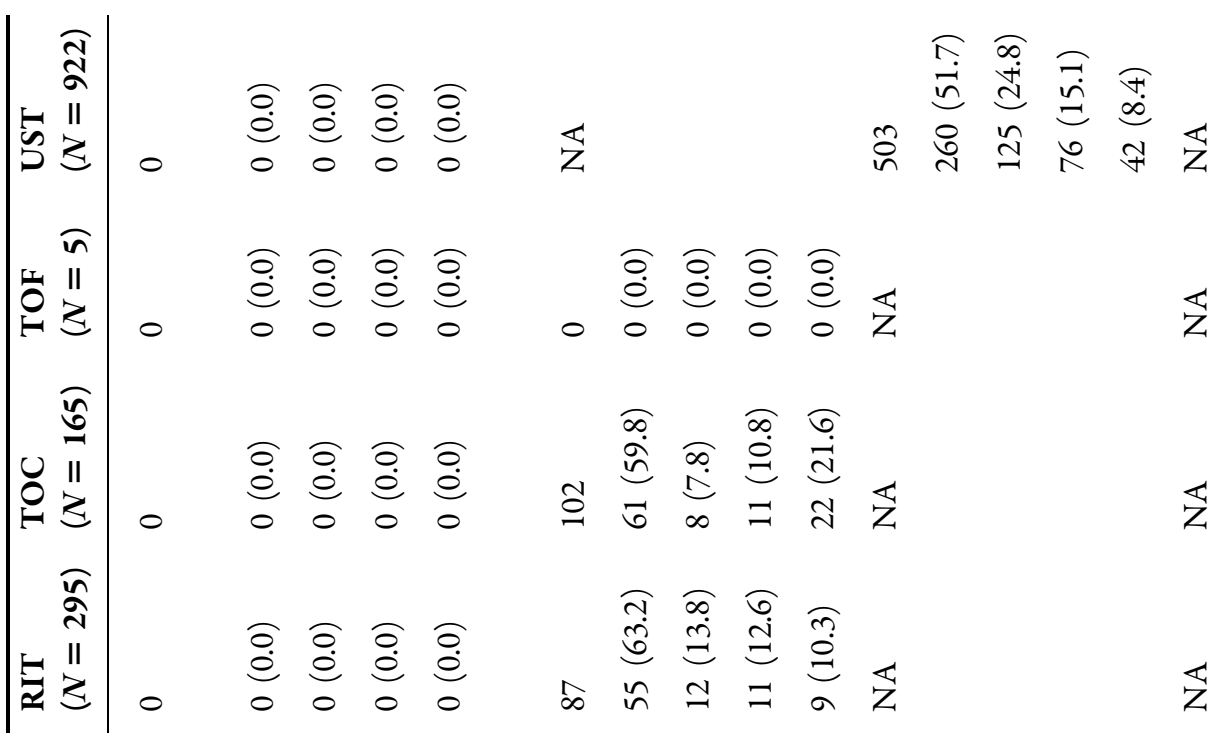

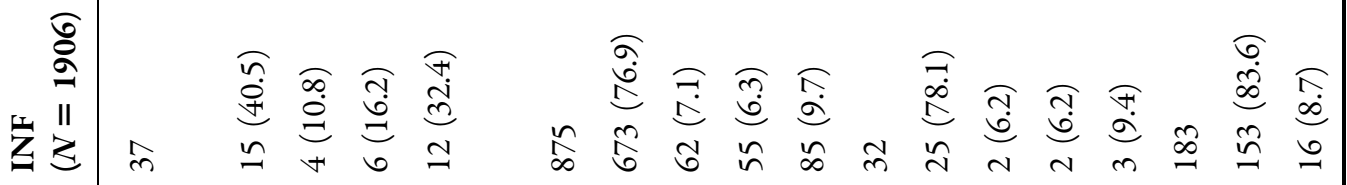

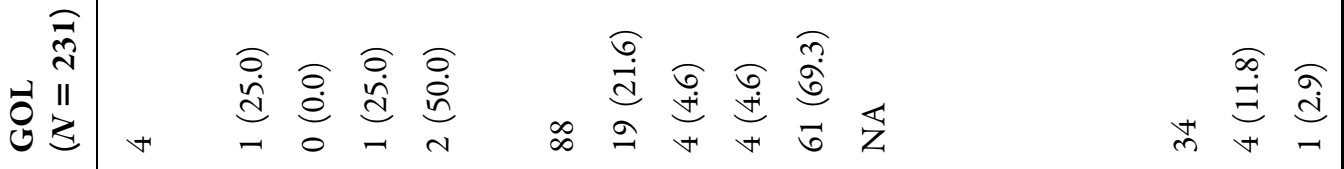

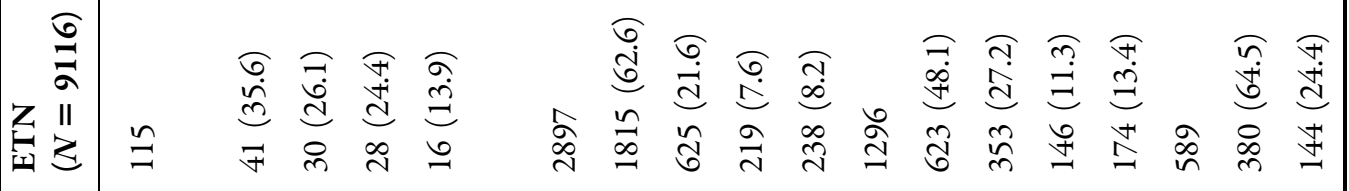

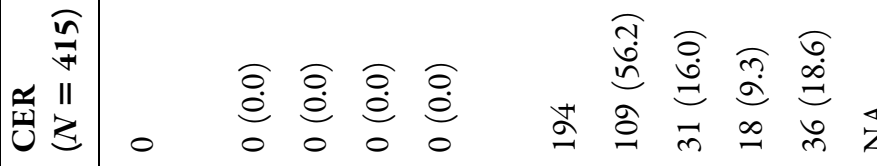

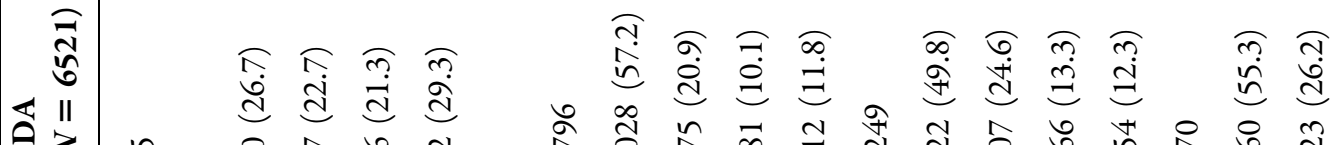

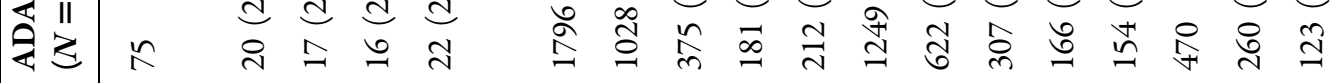

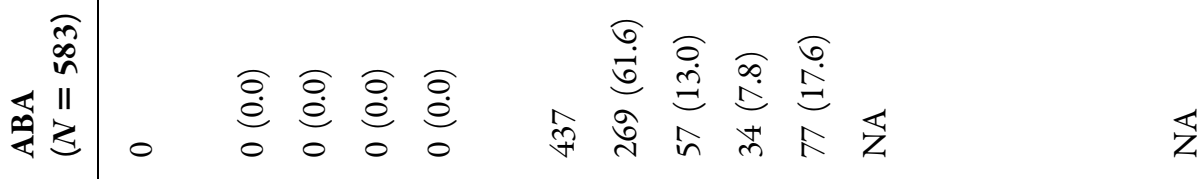

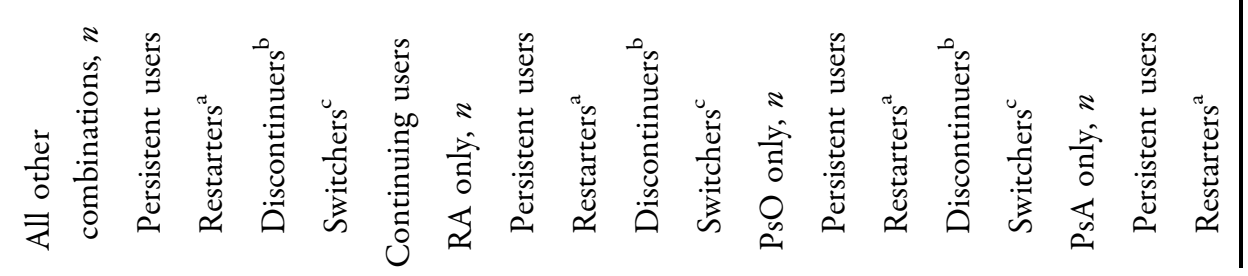




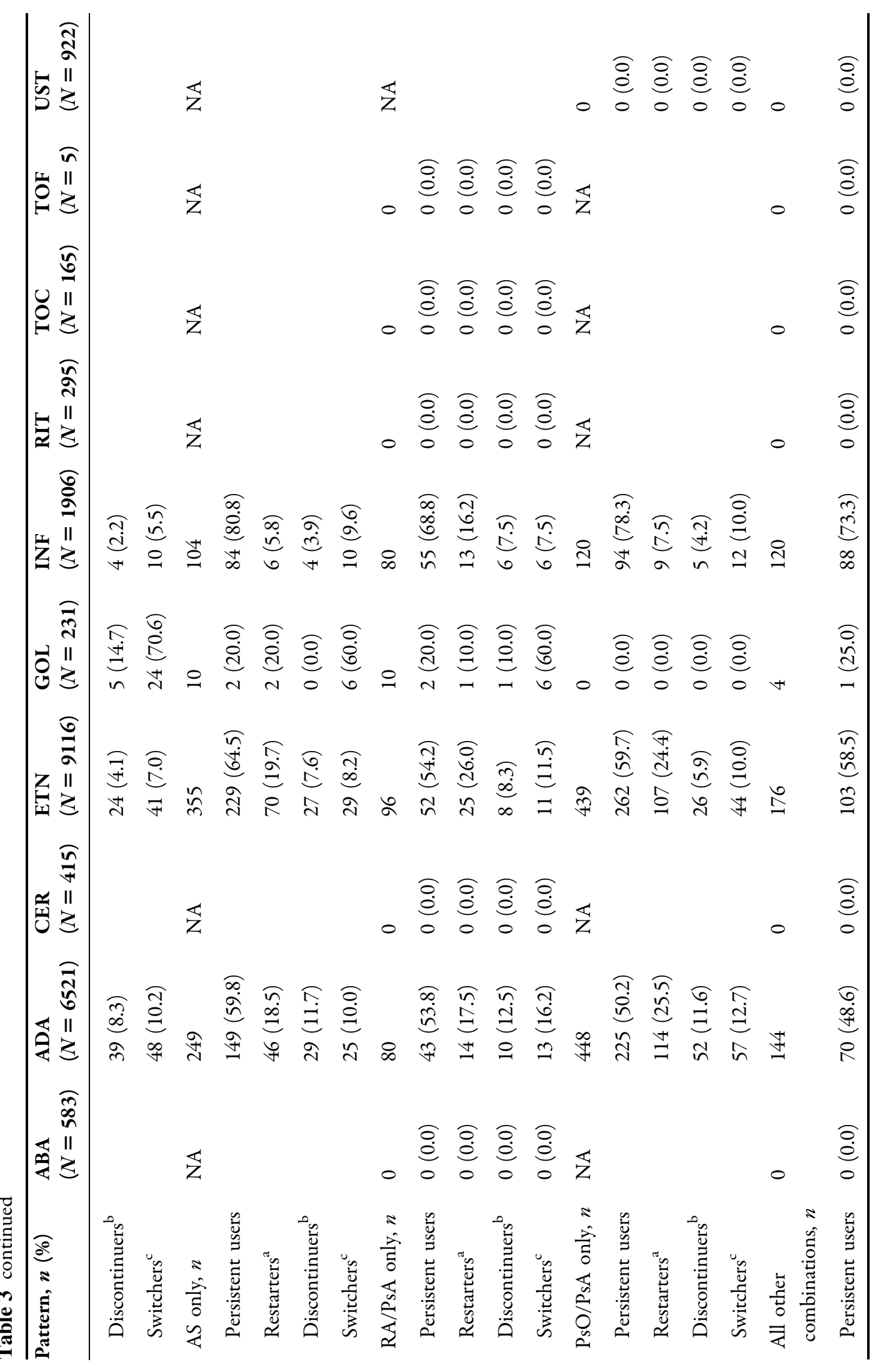




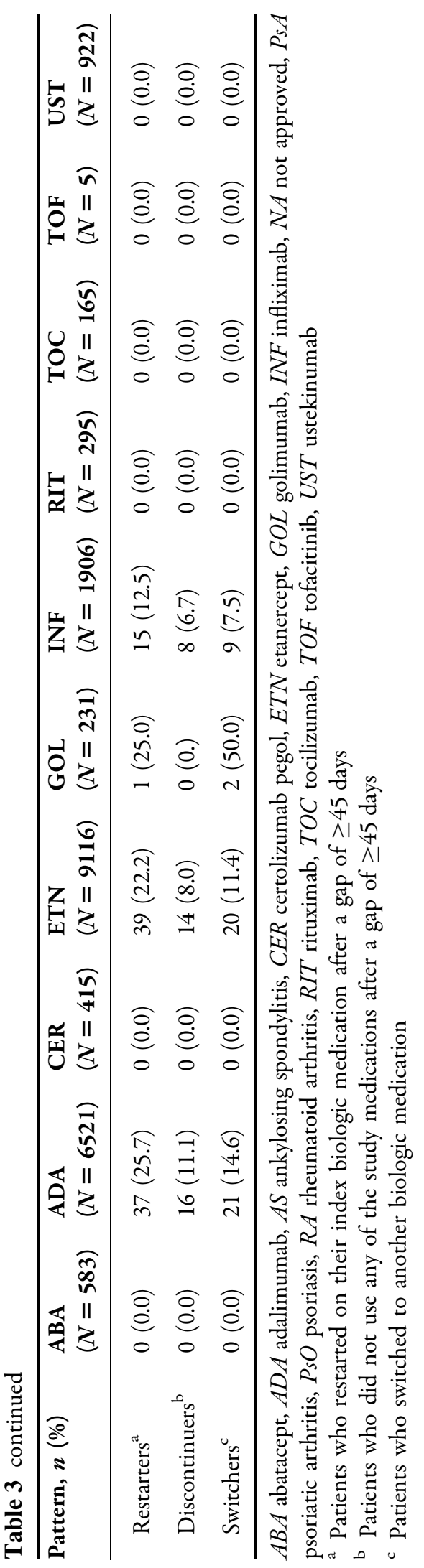

Patients who were new to therapy generally experienced lower biologic costs, though the decreased costs may be in part due to lower rates of persistence and higher rates of discontinuation without switch or restart. For patients with $\mathrm{PsO}$ only who were new to therapy, higher costs were seen in the first year of treatment compared to continuing users for etanercept and infliximab, most likely because these medications are recommended at higher loading doses (up to 12 weeks for etanercept and up to 6 weeks for infliximab) to facilitate rapid skin clearance. The rate of discontinuation without switch or restart for all patients with RA was lowest among patients treated with golimumab (4\%), infliximab (10\%), and etanercept (11\%) indicating that patients treated with these medications at index were less likely to have a drug-free period during the follow-up. While the current study examined the total cost of biologic treatment in a year, it did not examine other disease-related costs or clinical outcomes. Whether discontinuation of the index biologic led to a worsening of disease symptoms and greater downstream healthcare costs warrants further investigation.

Etanercept was the most commonly used medication in this study and in a prior study [9]. Similar to the prior study, newer biologics had equivalent or lower costs than etanercept for RA, PsA, and AS. The current study also confirms the observation that for biologics that are indicated for all four inflammatory diseases (etanercept, adalimumab, and infliximab), etanercept had the lowest annual acquisition and administration costs [9].

This study has inherent limitations associated with observational studies based on administrative claims. Specifically, while claims data indicate whether a prescription was filled, they do not indicate if the medication was used as prescribed or at all. This study included only 
patients who were enrolled in a commercial health plan; results of this analysis may, therefore, not be generalizable to outcomes in patient populations with government-sponsored health insurance (e.g., Medicare, Veterans Health Administration coverage, state Medicaid coverage) or uninsured populations. Results are also not generalizable to patients outside the US, as treatment guidelines and costs vary across countries. Additionally, claims data lack clinical data that may impact the interpretation of the results. Specifically, the duration of clinical benefit is assumed to be constant for each of the infused medications, which could lead to overestimates of persistence for certain medications. For example, the estimated duration of clinical benefit for the last infusion of infliximab may be too long, since the label-recommended dosing frequency could vary from every 8 weeks to every 4 weeks, which may impart bias by overestimating the clinical benefit for the last infusion. Similarly, the total duration of treatment for continuing users is unknown, leading to potential bias if it differs by index medication. Important clinical information such as reasons for treatment modifications (switch, restart, discontinuation), and clinical response to the biologic is unavailable in a claims database. Claims data also do not contain biometric information, such as patient weight and thus weight was not incorporated into dosage calculations for infused biologics. ICD-9-CM codes are proxies for actual physician diagnoses and can be affected by miscoding, overcoding, and undercoding. All variables may be subjected, to some extent, to data entry errors or errors due to rounding (for numerical variables). These errors, however, are unlikely to differ across groups or outcomes. Finally, this study calculated healthcare cost based on the observed dose and the associated WAC price, which does not take into account negotiations between health plans, manufacturer's rebates, or other price modifications that could impact the actual health plan and patient paid amounts.

\section{CONCLUSIONS}

The findings from this study indicate that there is considerable variability in the 1-year cost of biologic treatment between medications as well as between indications and in new or continuing patients. Etanercept was shown to have lower costs relative to adalimumab and infliximab across the indications evaluated here except among patients with PsO only. Of the medications included in this analysis, ustekinumab was the most expensive therapy for PsO. Newer agents may be associated with lower treatment cost; however, future research is needed to see if these estimates persist with expanded use of the more recently approved biologic.

\section{ACKNOWLEDGMENTS}

This study was funded by Immunex, a wholly owned subsidiary of Amgen Inc. and by Wyeth, which was acquired by Pfizer in October 2009. The article processing charges and open access fee for this publication were funded by Amgen Inc. All named authors meet the International Committee of Medical Journal Editors (ICMJE) criteria for authorship for this manuscript, take responsibility for the integrity of the work as a whole, and have given final approval of the version to be published. Editorial assistance in the preparation of this manuscript was provided by Dikran Toroser of Amgen Inc. and Julia R. Gage of Gage Medical Writing, LLC. Support for this assistance was funded by Amgen Inc. 
Disclosures. Benjamin Chastek, John White, and Damon Van Voorhis are employees of Optum (Eden Prairie, MN, USA). Derek Tang and Bradley S. Stolshek are employees of Amgen Inc. (Thousand Oaks, CA, USA).

Compliance with Ethics Guidelines. This article does not contain any new studies with human or animal subjects performed by any of the authors.

Open Access. This article is distributed under the terms of the Creative Commons AttributionNonCommercial 4.0 International License (http://creativecommons.org/licenses/by-nc/4. $0 /$ ), which permits any noncommercial use, distribution, and reproduction in any medium, provided you give appropriate credit to the original author(s) and the source, provide a link to the Creative Commons license, and indicate if changes were made.

\section{REFERENCES}

1. Helmick CG, Felson DT, Lawrence RC, et al. Estimates of the prevalence of arthritis and other rheumatic conditions in the United States. Part I. Arthritis Rheum. 2008;58:15-25.

2. Stern RS, Nijsten T, Feldman SR, Margolis DJ, Rolstad T. Psoriasis is common, carries a substantial burden even when not extensive, and is associated with widespread treatment dissatisfaction. J Investig Dermatol Symp Proc. 2004;9:136-9.

3. Gelfand JM, Gladman DD, Mease PJ, et al. Epidemiology of psoriatic arthritis in the population of the United States. J Am Acad Dermatol. 2005;53: 573.

4. Strand V, Sharp V, Koenig AS, et al. Comparison of health-related quality of life in rheumatoid arthritis, psoriatic arthritis and psoriasis and effects of etanercept treatment. Ann Rheum Dis. 2012;71: 1143-50.

5. Kotsis K, Voulgari PV, Drosos AA, Carvalho AF, Hyphantis T. Health-related quality of life in patients with ankylosing spondylitis: a comprehensive review. Expert Rev Pharmacoecon Outcomes Res. 2014;14:857-72.

6. Wu N, Lee YC, Shah N, Harrison DJ. Cost of biologics per treated patient across immune-mediated inflammatory disease indications in a pharmacy benefit management setting: a retrospective cohort study. Clin Ther. 2014;36:1231-41.

7. Howe A, Eyck LT, Dufour R, Shah N, Harrison DJ. Treatment patterns and annual drug costs of biologic therapies across indications from the Humana commercial database. J Manag Care Spec Pharm. 2014;20:1236-44.

8. Schabert VF, Watson C, Joseph GJ, Iversen P, Burudpakdee C, Harrison DJ. Costs of tumor necrosis factor blockers per treated patient using real-world drug data in a managed care population. J Manag Care Pharm. 2013;19:621-30.

9. Bonafede $M$, Joseph GJ, Princic N, Harrison DJ. Annual acquisition and administration cost of biologic response modifiers per patient with rheumatoid arthritis, psoriasis, psoriatic arthritis, or ankylosing spondylitis. J Med Econ. 2013;16:1120-8. 